\title{
¿Pueden las TIC mejorar los resultados académicos? Diseños formativos y didácticos con soporte TIC que mejoran los aprendizajes: el caso de los contenidos digitales de ortografía de Digital-Text
}

Can ICT improve academic outcomes? Training and didactic designs with ICT support that improve learning: a case study of digital spelling content in Digital-Text \author{
miquelpf@blanquerna.url.edu \\ Elena Sofía Ojando Pons \\ elenasofiaop@blanquerna.url.edu \\ Universitat Ramon Llull, España
}

Miquel Àngel Prats I Fernández ${ }^{1}$

\section{Resumen:}

El presente trabajo pretende verificar si la aplicación sistemática de determinadas metodologías didácticas con el apoyo de los libros de texto digitales y una formación adecuada al profesorado puede comportar mejoras significativas en los aprendizajes de los estudiantes y en su rendimiento académico. Concretamente se ha elaborado una propuesta de trabajo entorno a la ortografía catalana con materiales didácticos de libros digitales, dirigida a alumnos de primero de ESO, etapa

\begin{abstract}
:
This paper aims to verify whether the systematic application of certain teaching methods with the support of digital textbooks and adequately trained teachers can incur significant improvements in student learning and academic performance. Specifically, we developed a proposal to work with Catalan spelling with digital books and learning materials, aimed at students of first ESO (middle school), an educational stage that has made significant investment in technological equip-
\end{abstract}

1 Dirección para correspondencia (correspondence address):

Miquel Àngel Prats i Fernández. Facultad de Psicología, Ciencias de la Educación y del Deporte Blanquerna. Universitat Ramon Llull. C/ Císter, 24-34. 08022 Barcelona (España). 
¿Pueden las TIC mejorar los resultados académicos? Diseños formativos y didácticos con soporte TIC que mejoran los aprendizajes: el caso de los contenidos digitales de ortografía de Digital-Text

Miquel Àngel Prats I Fernández y Elena Sofía Ojando Pons

educativa donde se ha hecho una inversión importante en dotación tecnológica (aulas del proyecto eduCAT $1 \times 1$ ) y por lo tanto, se utilizan libros y material educativo digital.

En el estudio han participado 27 centros educativos de Cataluña. Concretamente, en 18 de estos centros -centros experimentales- se ha aplicado esta metodología y los otros 9 centros restantes -centros control - han trabajado los mismos contenidos sin utilizar recursos TIC.

Los resultados de la experimentación confirman que los alumnos del grupo experimental obtienen resultados significativamente mejores en los exámenes. Por lo tanto, en este estudio se pone de manifiesto que la debida formación al profesorado en medios digitales; la realización sistemática de determinadas actividades de aprendizaje con soporte TIC previamente planificadas y con un elevado grado de intencionalidad de refuerzo y repaso permite mejorar los aprendizajes y los resultados académicos de los estudiantes.

\section{Palabras clave:}

Material digital; libros digitales; metodologías didácticas y $\mathrm{TIC}$; rendimiento académico y TIC. ment (classrooms within the Educat $1 \times 1$ project). Therefore, digital books and educational materials are used.

The study involved 27 schools in Catalonia. Specifically, 18 of these centers (experimental centers) have applied the methodology; and the remaining 9 centers (control centres) have worked with the same content without ICT resources.

The results confirm that students in the experimental group obtained significantly better results in tests. Therefore, this study proves that appropriate training teachers in digital media; systematic use of pre-planned learning activities with ICT support as well as improved objectives regarding reinforcement and practice can improve students' learning and academic outcomes.

\section{Keywords:}

Digital media; digital books; teaching methodologies with ICT; academic performance with ICT.

\section{Résumé:}

Ce document vise à vérifier si l'application systématique de certaines méthodes d'enseignement avec le soutien de manuels numériques et d'enseignants correctement formés peuvent amener des améliorations significatives dans I'apprentissage des élèves et dans leur rendement scolaire. Plus précisément, nous avons développé une proposition de travail autour de l'orthographe catalane a l'aide du matériel dıapprentissage numérique, destiné aux étudiants de première année d/ESO. II s'agit en effet du stade de liéducation où un investissement important dans lıéquipement technologique (eduCAT projet $1 \mathrm{X} 1$ ) a été fait et dans laquelle les livres at d'autres matériaux éducatifs numériques sont utilisés. L'étude a porté sur 27 écoles en Catalogne. Plus précisément, 18 de ces centres ont appliqué cette méthodologie tandis que le 9 restants ont servi de contrôle et ont étudié le même contenu sans utiliser les ressources des TIC.

Les résultats expérimentaux confirment que les élèves du groupe test ont obtenu des résultats significativement meilleurs. Il apparait donc évident que les enseignants de formation appropriés dans les médias numériques; lıutilisation systématique de certaines activités diapprentissage avec lıaide de TIC spécifiquement élaborées a cette fin améliorent liapprentissage et le rendement scolaire des élèves lors des examens.

\section{Mots-clés:}

Médias numériques; livres numériques; les méthodes d'enseignement et les TIC; le rendement scolaire avec les TIC. 
¿Pueden las TIC mejorar los resultados académicos? Diseños formativos y didácticos con soporte TIC que mejoran los aprendizajes: el caso de los contenidos digitales de ortografía de Digital-Text Miquel Àngel Prats I Fernández y Elena Sofía Ojando Pons

Fecha de recepción: 6-1-2015

Fecha de aceptación: 8-6-2015

\section{Introducción}

Entre los meses de abril y junio de 2011 se llevó a cabo una investigación entre el grupo de investigación PSITIC (Pedagogía, Sociedad, Innovación y TIC) de la Facultad de Psicología, Ciencias de la Educación y del Deporte Blanquerna (Universidad Ramon Llull), el CETEI (Centro de Tecnologías Ituarte de la Fundación Joan XXIII) y el grupo de investigación Didáctica y Multimedia (DIM) con el objetivo de verificar si la aplicación sistemática de determinadas metodologías didácticas con el apoyo de los libros de texto digitales puede conllevar mejoras significativas en los aprendizajes de los estudiantes y en su rendimiento académico. Concretamente se elaboró una propuesta de trabajo entorno a la ortografía catalana con los materiales de la empresa Digital Text, dirigida a los alumnos de primero de ESO.

\section{La posible mejora de los aprendizajes con el soporte tecnológico}

La apuesta por la incorporación masiva de las tecnologías en las aulas puede suponer uno de los cambios metodológicos más disruptivos en toda la historia de la educación (Beetham \& Sharpe, 2007). La relevancia de este proceso de digitalización debe enmarcarse en la trascendencia educativa que supone introducir en la escuela las herramientas propias del siglo XXI. Estas herramientas posibilitan procesos de aprendizaje innovadores que, a su vez, también favorecen el cambio del rol del profesor que pasa a convertirse en un guía, que impulsa el trabajo colaborativo y entre iguales, y un aprendizaje más personalizado (Kozma, 2011).

Se trata de una decisión estratégica que debe conducir a cambios para lograr mejoras significativas de los resultados de los alumnos y detener la sangría del abandono escolar. Pero sólo el hecho de poner un portátil por alumno en el aula no quiere decir que el éxito educativo esté garantizado (Kozma, 2011). Esta evidencia ha quedado demostrada por la desproporción que ha habido entre la inversión en hardware tecnológico, acompañada de un tipo de formación aislada de su aplicación en 
¿Pueden las TIC mejorar los resultados académicos? Diseños formativos y didácticos con soporte TIC que mejoran los aprendizajes: el caso de los contenidos digitales de ortografía de Digital-Text

Miquel Àngel Prats I Fernández y Elena Sofía Ojando Pons

el aula que se ha hecho en los últimos diez años, y los resultados en los alumnos. Se trata de un cambio sistémico, que exige numerosos esfuerzos de entrada y un estrecho acompañamiento del profesorado, a la vez que una verdadera investigación aplicada de la evolución y resultados del proceso de implementación (Weston \& Bain, 2010).

Necesitaremos mayores competencias en herramientas científicas, matemáticas y para potenciar la creatividad, habilidad en la fluidez en el uso de las tecnologías de la información y de la comunicación; y en la adquisición de competencias como la capacidad de resolver situaciones complejas.

El sistema debe ayudar y facilitar estas transformaciones que permitan al profesorado educar en un entorno que potencie la inteligencia, la inteligencia social y la interconectividad. De esta manera seremos capaces de ofrecer al alumnado referentes y herramientas para la construcción de una ciudadanía verdaderamente democrática (Pedró, 2011).

Por un lado, la digitalización de contenidos está impulsando de manera decidida la labor de los editores de libros digitales, que están adecuando los contenidos a los nuevos formatos y deben incorporar los cambios necesarios para garantizar la calidad de los materiales didácticos. Los profesores, igualmente, deberán adaptar sus métodos de enseñanza a estas nuevas tecnologías y deberán aprovechar la existencia de otras tecnologías, como son las pizarras digitales, para optimizar la eficacia de las nuevas tecnologías. Se trata de una situación nueva, desconocida hasta ahora en los procesos de renovación pedagógica, y que pide un extraordinario cuidado en la evaluación de los resultados para garantizar la calidad del proceso de enseñanza y aprendizaje, como también un esfuerzo adicional de difusión de buenas prácticas y de intercambios de experiencias (Scheuermann \& Pedró, 2009). Aunque, el propio proyecto eduCAT $1 \times 1$, tal como se presenta y se define, no está asociado, en primera instancia, a ningún cambio metodológico específico.

En este sentido, son los equipos didácticos quienes deben definir el proyecto educativo de su centro, en una estrategia por fases en la que el paso del formato analógico (libro de texto, en papel) en formato digital (libro de texto digital) se hace primero, y el cambio hacia metodologías avanzadas de aprendizaje y enseñanza no es necesario que se haga simultáneamente, sino en los plazos que cada centro determine. En este sentido, el proyecto presupone que el cambio metodológico es mucho 
¿Pueden las TIC mejorar los resultados académicos? Diseños formativos y didácticos con soporte TIC que mejoran los aprendizajes: el caso de los contenidos digitales de ortografía de Digital-Text Miquel Àngel Prats I Fernández y Elena Sofía Ojando Pons

más fácil, una vez conectado al mundo digital (de Pablos, Area, Valverde y Correa, 2010).

Por otra parte, la decidida política de dar mayor autonomía a los centros educativos para precisar su proyecto educativo y, dentro de esta posibilidad, concretar los materiales digitales, las infraestructuras y las tecnologías necesarias para llevarlo a cabo, supone un alto reto para un sistema acostumbrado a pocos cambios (Ruíz, 2007).

\section{El contexto del proyecto Escuela 2.0 en Catalunya: eduCAT $1 \times 1$}

Esta investigación se llevó a cabo en el marco del proyecto eduCAT 1x1. Básicamente, el proyecto eduCAT $1 \times 1$, a pesar de tener su propia estrategia, por una parte, aprovechó los recursos que el Ministerio de Educación ponía a disposición de las comunidades autónomas mediante el proyecto de ámbito estatal Escuela 2.0, promulgado por el presidente del gobierno español , José Luís Rodríguez Zapatero, hace ya más de cuatro años; y, por otra parte, tiene como objetivo integrar las tecnologías de la información y de la comunicación (TIC) en los centros educativos de Cataluña y, en particular, en la etapa de secundaria.

Este proyecto, que se inició el curso 2009-2010 en los institutos de educación secundaria, tiene en cuenta el uso de un ordenador portátil por parte de cada alumno o alumna y la transformación de las actuales aulas en "aulas digitales" con acceso a Internet y a contenidos y recursos educativos en formato digital.

Los principales ejes del proyecto eduCAT $1 \times 1$ son:

1. Tecnologías digitales para el alumnado y docentes

Cada alumno dispone de un ordenador portátil personal homologado, cofinanciado por el Departamento de Educación y las familias. El profesorado debe tener a su disposición un ordenador portátil y el aula digital debe estar equipada con una pizarra digital interactiva (PDI).

2. Recursos educativos en formato digital

El Proyecto eduCAT 1x1 promueve la utilización de recursos educativos en formato digital que producen terceros, como vía para facilitar la incorporación de las tecnologías digitales a la práctica docente. Estos recursos educativos, los pueden producir editoriales y, por tanto, están sujetos al abono de derechos de uso, o por entidades o au- 
¿Pueden las TIC mejorar los resultados académicos? Diseños formativos y didácticos con soporte TIC que mejoran los aprendizajes: el caso de los contenidos digitales de ortografía de Digital-Text

Miquel Àngel Prats I Fernández y Elena Sofía Ojando Pons

tores de forma altruista, y ser en este caso gratuitos. El Departamento de Educación ofrece una dotación económica para cofinanciar la compra de los recursos educativos que los centros determinen por parte de las familias. En los dos primeros años del proyecto, esta cofinanciación ha sido de 30 euros para cada alumno y alumna.

El Proyecto eduCAT $1 \times 1$ no está asociado, en primera instancia, a ningún cambio metodológico específico. Son los equipos didácticos quienes deben definir el proyecto educativo de su centro, en una estrategia por fases en la que el paso del formato analógico (libro de texto, en papel) en formato digital (libro de texto digital) se hace primero, y el cambio hacia metodologías avanzadas de aprendizaje y enseñanza no es necesario que se haga simultáneamente, sino en los plazos que cada centro determine. El cambio metodológico es mucho más fácil una vez conectado al mundo digital.

3. Entornos virtuales de aprendizaje (EVA)

La gestión del entorno del aula y del aprendizaje individual se apoya en la utilización de un entorno virtual de aprendizaje (EVA), que viene a ser una especie de campus virtual del centro, que es a la vez una vía para la introducción de los cambios metodológicos que se mencionan más arriba. Los programas que integran un EVA, como los recursos educativos, los producen empresas dedicadas a esta actividad económica o grupos altruistas. El proyecto defiende la utilización de estos programas en la modalidad de servicio, a fin de librar a los centros de tareas de carácter tecnológico que está previsto que evolucionen hacia niveles de muy alta complejidad. En los centros públicos, también se cuenta con una dotación económica para la contratación de este tipo de servicios.

4. Recursos y servicios educativos que residen en Internet

El Proyecto eduCAT 1 $\times 1$ promueve que, tanto los recursos educativos como los programas que ayudan a gestionarlos, sean en Internet. Internet es una ventana a un mundo inacabable de recursos y servicios, y formarse para la vida en un mundo conectado es una parte muy importante del desarrollo de las competencias digitales que son indispensables en el mundo de hoy.

5. Conectividad en las aulas

Las aulas eduCAT $1 \times 1$ tienen conexión a Internet mediante una red específica para todos los ordenadores que suministra el ancho de banda necesario para el trabajo de alumnos y docentes. La 
¿Pueden las TIC mejorar los resultados académicos? Diseños formativos y didácticos con soporte TIC que mejoran los aprendizajes: el caso de los contenidos digitales de ortografía de Digital-Text Miquel Àngel Prats I Fernández y Elena Sofía Ojando Pons

conexión de los ordenadores se hace normalmente mediante una red local con tecnología Wi-Fi. Los ordenadores de los alumnos acceden al entorno virtual de aprendizaje y en Internet bajo la supervisión de los docentes.

6. Formación del profesorado

El Departamento de Educación dispone de una oferta específica de formación presencial y no presencial por el Proyecto eduCAT 1x1, centrada en los aspectos tecnológicos y metodológicos necesarios para integrar las herramientas y los recursos digitales en la práctica docente. También ofrece acompañamiento formativo virtual a los docentes y asesoramiento a los docentes adscritos al proyecto. Se prevé que en 4 años (2014-2015) el proyecto se extienda a todos los alumnos de secundaria y el ciclo superior de educación primaria a partir del equipamiento de los centros (cada centro dispone de ordenadores, sistemas, de electrificación de las aulas, pizarras digitales y conectividad $\mathrm{Wi}$-Fi), la formación del profesorado (formación y acompañamiento al profesorado ya los centros educativos), los recursos educativos digitales y, especialmente, la plataforma Àtria (los recursos educativos digitales son la alternativa al libro de texto en papel y la plataforma digital Àtria, el lugar que suministra estos recursos).

\section{Etapa educativa}

Se seleccionó la etapa educativa de la ESO, ya que, era en esa precisa etapa donde se había realizado fuertes inversiones en dotaciones e infraestructuras tecnológicas en las aulas en relación al proyecto eduCAT $1 \times 1$. Por ello, muchos centros estaban utilizando ya los libros digitales en la etapa de secundaria obligatoria, incluso algunos de los centros participantes también incluyeron grupos de sexto de primaria.

\section{Aprendizajes objeto de estudio}

Se buscaron elementos curriculares importantes, muy concretos y fácilmente evaluables de manera objetiva. $Y$ de todos ellos, finalmente se escogió la ortografía catalana. De hecho, determinados contenidos de la 
¿Pueden las TIC mejorar los resultados académicos? Diseños formativos y didácticos con soporte TIC que mejoran los aprendizajes: el caso de los contenidos digitales de ortografía de Digital-Text

Miquel Àngel Prats I Fernández y Elena Sofía Ojando Pons

ortografía catalana: la sílaba tónica, palabras agudas, planas y esdrújulas, las normas de acentuación, el acento diacrítico, la vocal neutra, la b y la v, la g y la j, la x y los dígrafos ix/tx/ig, s sorda y s sonora (s, ss, z, c, ç).

Así pues, aunque se considera que el uso adecuado de las TIC en la educación podría mejorar el desarrollo de diversas competencias básicas por parte del alumnado, esta experimentación se centró únicamente en el aprendizaje de la ortografía catalana, un aprendizaje completamente memorístico, dado que consiste en asimilar una normativa arbitraria (incluyendo la correlación básica entre unos sonidos y unas grafías), unas excepciones y unos casos.

\section{Instrumentos TIC necesarios}

Se utilizaron los libros de texto multimedia interactivos de Digital-Text y la plataforma Eleven, herramientas desarrolladas expresamente para mejorar los procesos de enseñanza y aprendizaje a través de las TIC (ordenadores e Internet) y que se utilizan en muchas escuelas que participaron en el proyecto eduCAT $1 \times 1$.

Un aspecto diferencial de las TIC del que sacamos provecho en esta experimentación fue la capacidad que proporcionan las TIC de gestionar la información, que se traduce en la posibilidad de programar automáticamente las tareas (actividades) que el alumnado debe cumplir, realizar un seguimiento exhaustivo y personalizado de su avance, e intervenir de forma individualizada en caso necesario. Y todo ello sin un incremento significativo del tiempo de dedicación docente.

Por otra parte, también se consideró que el alumnado realizaría, como actividades de repaso o refuerzo, muchos más ejercicios con las TIC de los que habitualmente se realizan en los entornos de trabajo tradicionales (sin TIC), debido tanto a la prescripción de tareas de los docentes como a un incremento en su motivación por el feedback automático e inmediato que proporcionan las actividades en estos entorno.

\section{Centros participantes}

En total participaron 27 centros educativos de Cataluña repartidos casi al $50 \%$ entre centros públicos y centros privados/concertados. 
¿Pueden las TIC mejorar los resultados académicos? Diseños formativos y didácticos con soporte TIC que mejoran los aprendizajes: el caso de los contenidos digitales de ortografía de Digital-Text Miquel Àngel Prats I Fernández y Elena Sofía Ojando Pons

La mitad de los centros participantes (centros experimentales) aplicaron esta metodología y los otros (centros control) trabajaron los mismos contenidos sin utilizar recursos TIC.

Para facilitar la puesta en marcha de la investigación, que se decidió hacer el mes de marzo, la selección de los centros se hizo de manera intencional, entre centros innovadores y participantes en el proyecto eduCAT $1 \times 1$ que ya habían participado en otras experimentaciones del CETEl, o del grupo de investigación PSITIC, del grupo de investigación DIM o de Digital-Text. Estos centros experimentales, además son centros usuarios de los contenidos de Digital Text.

Un aspecto clave de la investigación era asegurar que los centros experimentales, además de disponer de las herramientas de software adecuadas (en este caso los contenidos educativos interactivos y multimedia de Digital-Text), disponían de los equipamientos tecnológicos (ordenadores, conectividad...) necesarios y de un profesorado con la formación adecuada para hacer un buen uso didáctico de estos recursos.

Se procuró que los grupos control y experimentales tuvieran representantes equivalentes en relación al tipo de escuela, nivel económico, geografía, número de alumnos, etc.

\section{Diseño de la investigación}

Tras un examen inicial en papel (material analógico) igual para todos los alumnos, los alumnos de los centros control trabajaron los contenidos de la ortografía catalana de la manera que determinaron sus profesores dentro de la asignatura de Lengua Catalana y Literatura, pero sin utilizar los ordenadores.

El profesorado de los grupos control, aunque no podían utilizar herramientas digitales para preparar a los alumnos, fue informado de la metodología que se aplicaría a los grupos experimentales, y se comprometió a trabajar, un mínimo de horas en clase, la ortografía objeto de este estudio. Salvo el uso de las TIC, el profesorado podía utilizar los recursos y las estrategias de motivación que considerara más oportunas.

Por su parte, los alumnos de los centros experimentales aplicaron la metodología diseñada por los investigadores, y pudieron trabajar con los 
¿Pueden las TIC mejorar los resultados académicos? Diseños formativos y didácticos con soporte TIC que mejoran los aprendizajes: el caso de los contenidos digitales de ortografía de Digital-Text

Miquel Àngel Prats I Fernández y Elena Sofía Ojando Pons

contenidos de Digital-Text en los ordenadores de la escuela y también desde casa, dado que esta metodología se basa sobre todo en el trabajo individualizado que se puede conseguir gracias a las TIC.

El profesorado de los grupos experimentales se comprometió a seguir al $100 \%$ la metodología y programación indicada por los investigadores. Además, podía utilizar también otros recursos y las estrategias de motivación que considerara más oportunas.

Al final de la etapa de trabajo que duró unos dos meses aproximadamente, todos los alumnos hicieron un nuevo examen en papel, el examen final, con el fin de poder comprobar la mejora en sus aprendizajes.

Las pruebas inicial y final fueron elaboradas por un equipo independiente de especialistas en lengua catalana. Los profesores no conocieron el contenido hasta que fueron entregadas a los alumnos. Los exámenes tenían preguntas de respuesta múltiple y preguntas que exigen una sola palabra o letra de respuesta (para evitar la subjetividad del corrector). Las pruebas también incluían una parte inicial con dictados de palabras y frases. Cabe destacar que las pruebas inicial y final contenían las mimas preguntas pero en un orden diferente.

Generalmente, fue el propio profesorado participante quien se encargó de la corrección de las pruebas, según los criterios indicados por los investigadores, y de la introducción de estos datos en una tabla de resultados.

Al terminar la experimentación, el profesorado participante también Ilenó un pequeño cuestionario especificando el tiempo dedicado a preparar clases, corregir ejercicios, etc.

A partir de los datos proporcionados en la prueba inicial y la final, se pudo comprobar la mejora en la ortografía dentro de un mismo grupo, considerando el conjunto de los grupos control y el conjunto de los grupos experimentales. Se identificaron las diferencias entre la mejora lograda por los grupos control en comparación con la mejora lograda por los grupos experimentales.

\section{Metodología y planificación utilizada por los centros experimentales}

Los profesores de los centros experimentales podían dedicar el tiempo que considerasen oportuno para explicar y trabajar los contenidos de or- 
¿Pueden las TIC mejorar los resultados académicos? Diseños formativos y didácticos con soporte TIC que mejoran los aprendizajes: el caso de los contenidos digitales de ortografía de Digital-Text Miquel Àngel Prats I Fernández y Elena Sofía Ojando Pons

tografía catalana en clase, pero después era preceptivo que los alumnos realizaran con los contenidos de Digital-Text las actividades previstas en la programación.

\section{Planificación del trabajo a realizar}

- Primera semana de abril: Evaluaciones iniciales de los alumnos y presentación al profesorado del trabajo a realizar.

Todos los alumnos realizaron un mismo examen inicial, en formato papel y elaborado por especialistas de lengua catalana en torno a los contenidos objeto del estudio, para determinar sus conocimientos iniciales sobre la ortografía catalana.

La prueba tenía dos partes: dictado y preguntas tipo test.

- Abril/junio: Periodo de trabajo sistemático con los alumnos de los contenidos de la ortografía catalana.

Los grupos experimentales utilizaron tecnologías para trabajar la ortografía de acuerdo con la metodología propuesta por los investigadores (e hicieron buena parte del trabajo en casa). Y los grupos control lo hicieron siguiendo los métodos tradicionales y de acuerdo con las indicaciones de sus profesores.

- Principio de junio: Evaluación final de los alumnos.

Todos los alumnos realizaron un mismo examen final, en formato papel, para determinar sus conocimientos finales sobre la ortografía catalana y comprobar la mejora en sus aprendizajes. El ejercicio tenía una dificultad idéntica a la del examen inicial, y también incluía: dictado y preguntas tipo test.

El profesorado también respondió un breve cuestionario especificando el tiempo dedicado a preparar clases, corregir ejercicios, etc.

- Junio/julio: Corrección de las evaluaciones finales, análisis de los resultados y presentación de la memoria de la investigación. 
¿Pueden las TIC mejorar los resultados académicos? Diseños formativos y didácticos con soporte TIC que mejoran los aprendizajes: el caso de los contenidos digitales de ortografía de Digital-Text

Miquel Àngel Prats I Fernández y Elena Sofía Ojando Pons

\section{Resultados obtenidos}

- Prueba inicial. La media de errores de los grupos experimental y control fueron prácticamente iguales (42,9 el grupo experimental y 42,4 el grupo control), por lo que podemos considerar los grupos bastante similares.

- Prueba final. En los centros experimentales, la media de errores ha bajado a 34,1 , en tanto que los centros control siguen con una media de 40,8

Es decir, en los centros experimentales hemos tenido una reducción de los errores del $\mathbf{2 0 , 5} \%$ en tanto que en los centros control la mejora ha sido sólo del 3,9\%

En la siguiente tabla se pueden ver todos los datos y comprobar las mejoras de los dos grupos en la prueba del dictado y en la prueba del test.

Tabla 1. Resultados de las pruebas de dictado y test en los grupos control y experimental.

\begin{tabular}{lllllll}
\hline Promedio & Inicio & Final & \% menos de errores & Desvest & Inicio & Final \\
\hline Dict c & 10.5 & 10.1 & 3.7 & Control & 3.1 & 3.4 \\
Dict e & 9.5 & 7.8 & 17.1 & Exp & 3.2 & 2.6 \\
Test c & 32.0 & 30.7 & 4.2 & Control & 9.3 & 11.1 \\
Test e & 33.4 & 26.2 & 21.5 & Exp & 8.2 & 8.3 \\
Total c & 42.4 & 40.8 & 3.9 & Control & 11.8 & 14.3 \\
Total e & 42.9 & 34.1 & 20.5 & Exp & 11.0 & 10.6 \\
\hline
\end{tabular}

$*$ Dict $=$ errores en la prueba de dictado. Test $=$ errores en la prueba de test. Total $=$ Suma de errores de las dos pruebas. $\mathrm{c}=$ grupos control. $\mathrm{e}=$ grupos experimentales.

El análisis estadístico mediante la prueba $T$ de Student para datos apareados indica que las diferencias entre los resultados al inicio y al final de los grupos control no son estadísticamente significativas. En cambio, las diferencias observadas en los grupos experimentales sí son fuertemente significativas (con valores de $\mathrm{p}$ inferiores al 0.001 en todos los casos) tal y como podemos ver la figura siguiente. 
¿Pueden las TIC mejorar los resultados académicos? Diseños formativos y didácticos con soporte TIC que mejoran los aprendizajes: el caso de los contenidos digitales de ortografía de Digital-Text Miquel Àngel Prats I Fernández y Elena Sofía Ojando Pons

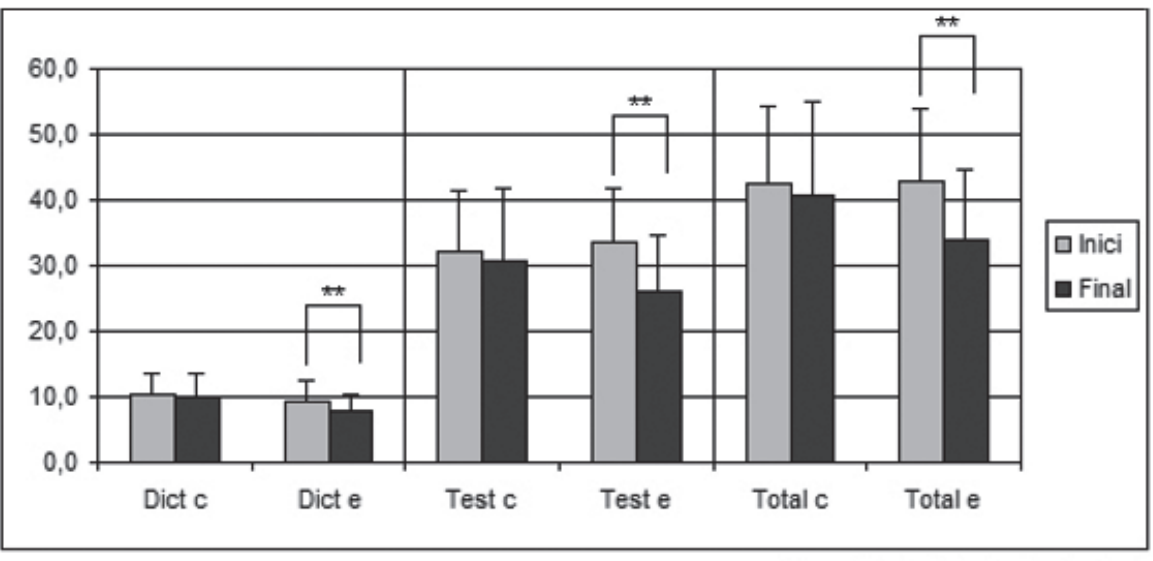

* $p<0,001$ (t-student paired)

Figura 1. Diferencias entre los resultados de las pruebas inicial y final de todos los centros.

\section{- Cuestionario del profesorado}

Como se puede observar en el siguiente gráfico el número de sesiones de clase dedicadas a trabajar la ortografía catalana ha sido bastante similar, aunque los centros control han dedicado un poco más de tiempo.

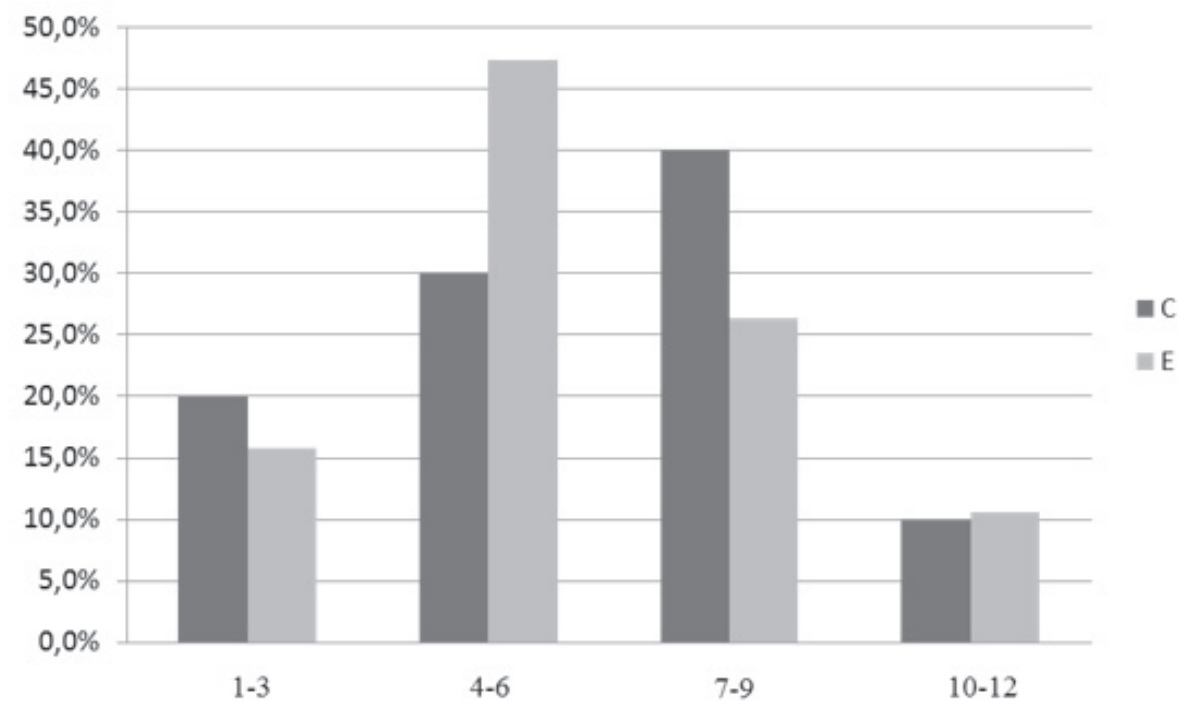

Figura 2. Gráfico con el número de sesiones de clase. 
¿Pueden las TIC mejorar los resultados académicos? Diseños formativos y didácticos con soporte TIC que mejoran los aprendizajes: el caso de los contenidos digitales de ortografía de Digital-Text

Miquel Àngel Prats I Fernández y Elena Sofía Ojando Pons

Los profesores de los grupos experimentales también han necesitado mucho menos tiempo para preparar actividades y corregir fuera del aula (ya que han utilizado los ejercicios auto-correctivos de Digital-text). El siguiente gráfico muestra las diferencias.

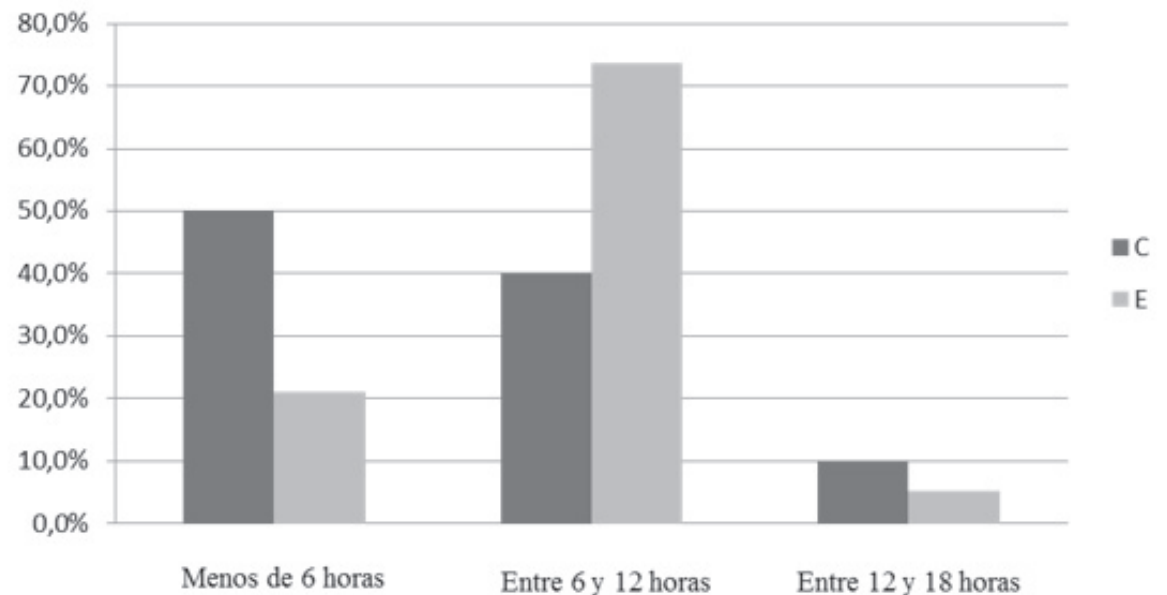

Figura 3. Gráfico con las horas dedicadas por el profesorado fuera del aula.

Según las apreciaciones del profesorado, el gráfico siguiente muestra las horas que sus alumnos han trabajado con los ejercicios de ortografía fuera del aula. Se pone de manifiesto que los alumnos experimentales han trabajado más tiempo en su casa.

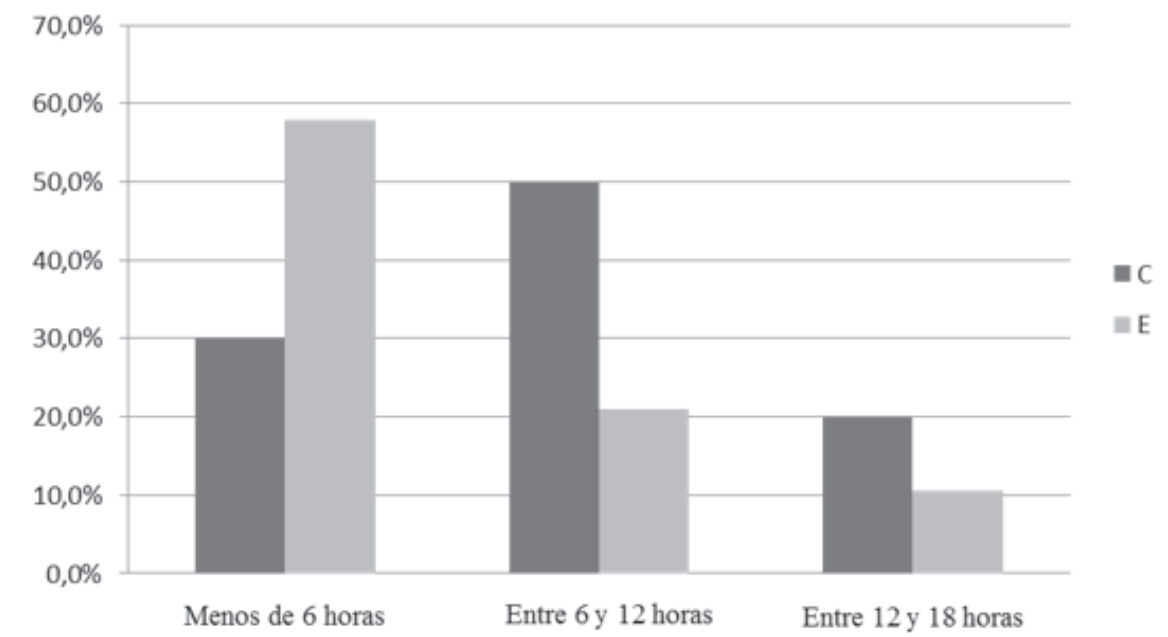

Figura 4. Gráfico con las horas dedicadas por el alumnado a los ejercicios de ortografía fuera del aula. 
¿Pueden las TIC mejorar los resultados académicos? Diseños formativos y didácticos con soporte TIC que mejoran los aprendizajes: el caso de los contenidos digitales de ortografía de Digital-Text Miquel Àngel Prats I Fernández y Elena Sofía Ojando Pons

Y finalmente el último gráfico muestra que el volumen de ejercicios realizados por los alumnos experimentales ha sido mucho más elevado que los ejercicios realizados por los alumnos del grupo de control.

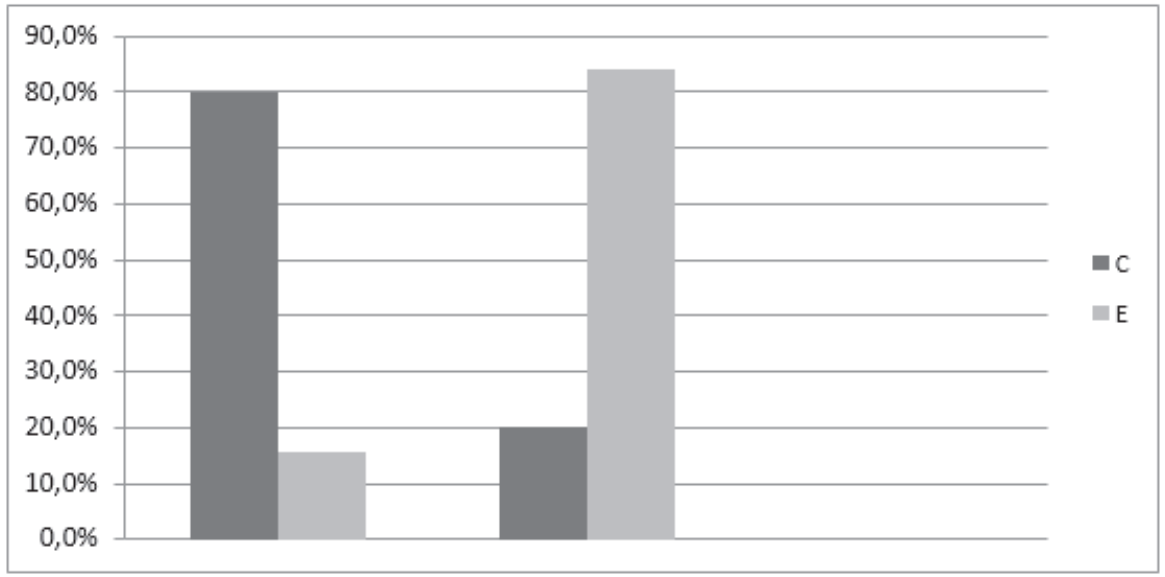

Figura 5. Gráfico con el volumen de ejercicios realizados por el alumnado (estimación del profesorado).

\section{Conclusiones}

Las principales conclusiones de este estudio son claras: los alumnos que han utilizado de manera sistemática (según la metodología y planificación previamente establecida con la adecuada formación del profesorado) los contenidos didácticos digitales (en este caso los ejercicios de ortografía de Digital-Text) han obtenido una mejora significativa de sus aprendizajes y rendimiento académico, con una media del 20,4\%. Los grupos control han obtenido solamente una pequeña mejora $(3,9 \%) \sin$ significatividad estadística.

Y esto ha sido así, porque los alumnos de los grupos experimentales han trabajado más, es decir, han realizado muchos más ejercicios (de refuerzo y repaso) de los que habitualmente hacen los alumnos cuando se utilizan las metodologías tradicionales (sin TIC) por su demostrada motivación frente la naturaleza interactiva de las herramientas TIC.

Por otra parte, destacamos que con el apoyo del ordenador y los ejercicios auto-correctivos, todo ello no ha supuesto un trabajo extra significativo por parte del profesorado, sino más bien lo contrario. Además, 
¿Pueden las TIC mejorar los resultados académicos? Diseños formativos y didácticos con soporte TIC que mejoran los aprendizajes: el caso de los contenidos digitales de ortografía de Digital-Text

Miquel Àngel Prats I Fernández y Elena Sofía Ojando Pons

las TIC han podido aportar la información del progreso (trazabilidad) del aprendizaje de cada alumno de manera personalizada.

\section{Referencias bibliográficas}

Beetham, H. i Sharpe, R (ed.). (2007). Rethinking Pedagogy for a Digital Age: designing and delivering e-learning. Abingdon: Routledge.

Consell Superior d'Avaluació del Sistema Educatiu. (2009). Les TIC a I'ESO. Resultats i conclusions del estudi SITES 2006 a Catalunya. Col/lecció Informes d'Avaluació, núm. 13.

de Pablos, J.; Area, M.; Valverde, J. i Correa, J.M. (coord.). (2010). Políticas educativas y buenas prácticas con TIC. Barcelona: Graó.

Fornell, R. i Vivancos, J. (2010). El Pla TAC de centre. Col·lecció TAC-1.

Gordó, G. (2010). Centros educativos: ¿islas o nodos? Los centros como organizaciones red. Barcelona: Graó.

Harasim, L.; Hiltz, S. R.; Turoff, M. i Teles, L. (2000). Redes de aprendizaje. Guía para la enseñanza y el aprendizaje en red. Barcelona: Gedisa.

Gee, J.P.; Shaffer, D.W.(2010). Looking where the light is bad: Video games and the future of assessment. Epistemic Games Group Working Paper 2010-12.

Kozma R. B. (2011). A Framework for ICT Policies to Transform Education. A: Transforming Education: The Power of ICT Policies (pp.19-36). París: UNESCO.

Weston, M.E. \& Bain, A. (2010). The End of Techno-Critique: The Naked Truth about 1:1. Laptop Initiatives and Educational Change. Journal of Technology, Learning and Assessment. 8(6).

Mominó, J., Sigalés, C., Meneses, J. (2008). L'escola a la societat xarxa. Barcelona: Ariel.

N. Law, W.J. Pelgrum, T. Plomp (ed.). (2008). Pedagogy and ICT use. In schools around the world. Findings from the IEA SITES 2006 study. CERC Studies in Comnparative Education 23. Springer \& Comparative Education Research Center - The University of Hong Kong.

OCDE. (2011). Students on Line: Reading and Using Digital Information, París: Publicacions de l'OCDE.

Pedró, F. (2011). Tecnología y Escuela: Lo que funciona y porqué. Documento básico.

Ruiz Tarragó, F. (2007). Vint-i-cinc anys de polítiques d'integració de les TIC als centres docents de Catalunya. A Mominó, J. M. \& Sigalés, C. (directors) L'escola a la societat xarxa. Projecte Internet Catalunya (dir. M. Castells).

Ruiz, F. (2007). La nueva educación. Madrid: LID.

Scheuermann, F. i Pedró, F. (ed.) (2009). Assessing the effects of ICT in education. Indicators, criteria and benchmarks for international comparisons. Luxemburg: Oficina de Publicacions de la Unió Europea.

Sigalés, C. et al. (2007). L'Escola a la Societat Xarxa. Internet a l'educació primària i secundària. Informe de recerca Vo.1, UOC.

UNESCO. (2008). Estándares de competencia en TIC para docentes. 
¿Pueden las TIC mejorar los resultados académicos? Diseños formativos y didácticos con soporte TIC que mejoran los aprendizajes: el caso de los contenidos digitales de ortografía de Digital-Text Miquel Àngel Prats I Fernández y Elena Sofía Ojando Pons

\section{Notas y/o Agradecimientos}

Este estudio se realizó conjuntamente con la codirección del Dr. Pere Marqués del Grupo de investigación Didáctica y Multimedia (DIM) de la Universidad Autónoma de Barcelona (UAB) sin el cual no hubiera sido posible.

También, cabe destacar la colaboración del CETEI Fundación Joan XXIII y del grupo de investigación PSITIC (Pedagogía, Sociedad, Innovación y TIC) de la Facultad de Psicología, Ciencias de la Educación y del Deporte Blanquerna (Universidad Ramon Llull), grupo al cual pertenecen los autores de este artículo. 
